
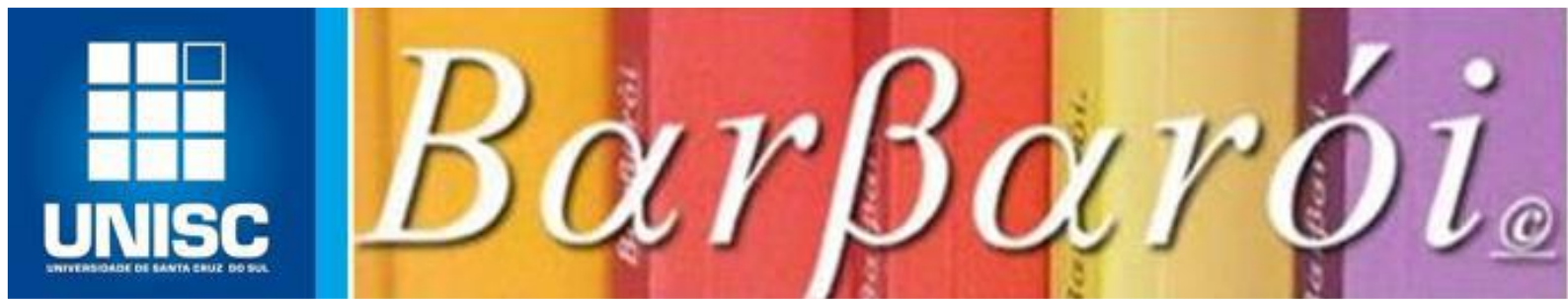

\title{
QUALIDADE DE VIDA EM SUJEITOS COM LESÃO MEDULAR: UMA REVISÃO SISTEMÁTICA
}

\author{
DOI: http://dx.doi.org/10.17058/barbaroi.v0i58.15801 \\ $*$
}

\author{
Carolina Antunes \\ Universidade do Feevale - Brasil \\ Marcus Levi Lopes Barbosa \\ Universidade do Feevale - Brasil \\ Gustavo Roese Sanfelice \\ Universidade do Feevale - Brasil \\ Jacinta Sidegum Renner \\ Universidade do Feevale - Brasil \\ Gabriel Feiten \\ Universidade do Feevale - Brasil
}

\section{Resumo}

O objetivo desse estudo é analisar e investigar a qualidade de vida em pessoas com lesão medular, através de uma revisão sistemática. Buscaram-se os principais trabalhos publicados no período de 2006 a 2019, nas bases de dados CAPES e Scielo. Todos os estudos utilizaram medidores quantitativos sobre qualidade de vida, os quais são: o WHOQOL-Bref e o SF-36. Os resultados de forma geral mostraram que o sujeito com lesão medular avalia sua qualidade de vida como boa e sua saúde de forma geral satisfatória. O domínio físico e do meio ambiente foram os domínios mais citados nas pesquisas como um fator negativo, sendo ele relacionado com a questão da acessibilidade. Verificou-se que estudos futuros são de extrema importância, para compreender a qualidade de vida desses indivíduos, assim como para a melhoria de políticas públicas para questão de acessibilidade e autonomia desses sujeitos.

Palavras-chave: Lesão Medular. Pessoas com deficiência. Qualidade de Vida.

\section{Introdução}

Os casos de lesão medular vem aumentando e, com isso, tornam-se um problema de saúde pública no Brasil (CASALIS, 2003; CAMPOS et al., 2008). Conforme França et al. (2013), há em média 7.000 casos de sujeitos com lesão medular traumática por ano. Os dados 
apontam que a maioria são homens solteiros, que vivem em áreas urbanas e com idade entre 18 e 35 anos (COURA et al., 2012; VALL; BRAGA; ALMEIDA, 2006). Pesquisas apontam que, em $80 \%$ dos casos, as lesões medulares são traumáticas, causadas por arma de fogo, quedas, mergulhos e acidentes automobilísticos e, nos $20 \%$ restantes, são lesões não traumáticas caracterizadas por tumores, doenças vasculares, degenerativas ou infecciosas (MEDOLA et al., 2009; VENTURINI; DECESARO; MARCON, 2006).

Por constituírem casos de grande gravidade e irreversíveis, é preciso olhar para esses sujeitos cuja vida sofre mudanças de forma instantânea, com modificações corporais, psicológicas e sociais, o que exige a retomada de sua autonomia para tarefas antes simples do dia a dia (BERTO; BARRETO, 2011). Sendo assim, o tema desse trabalho é a qualidade de vida em lesionados medulares.

Um dos fatores mais impactantes na lesão medular é a falta de acessibilidade. O acesso a muitos locais é considerado inadequado, mesmo existindo leis que regulamentam esse direito, como o acesso a rampas em lugares públicos, inclusive em banheiros. Na prática, isso nem sempre acontece. A acessibilidade não corresponde apenas à participação do sujeito com deficiências em atividades, mas a um processo de transformação do ambiente (MANZINI, 2006). Com frequência, é possível que o lesionado medular enfrente momentos de estresse ao sair de casa, pela necessidade de auxílio de terceiros para essas questões, o que afeta diretamente sua qualidade de vida em diversos fatores.

A Organização Mundial da Saúde define "qualidade de vida" como: "a percepção do indivíduo de sua posição na vida no contexto da cultura e sistema de valores nos quais ele vive e em relação aos seus objetivos, expectativas, padrões e preocupações" (THE WHOQOL GROUP, 1994 apud FLECK, 2000, p. 179).

A qualidade de vida está relacionada à capacidade do indivíduo em perceber elementos que a sociedade considera como padrões de bem-estar e conforto, baseados nas experiências e nos valores individuais e coletivos (MINAYO; HARTZ; BUSS, 2000). Segundo Almeida, Gutierrez e Marques (2012), ela reflete o interesse do homem pela vida e configura um objeto de desejo da sociedade, ainda que circunscrito à subjetividade de cada indivíduo que a busca, por meio do seu esforço e dedicação. Uma boa qualidade de vida gera condições para que as pessoas possam desenvolver ao máximo as suas potencialidades, atingindo índices elevados de felicidade, liberdade, inserção social e realização pessoal, além da satisfação de suas necessidades (MINAYO; HARTZ; BUSS, 2000; MOREIRA, 2001).

A compreensão dos fatores relacionados à qualidade de vida vai depender do conhecimento do sujeito em relação ao ambiente no qual ele vive, às suas interações com a Barbarói, Santa Cruz do Sul, n. 58, p.<141-153>, jan/jun 2021 
sociedade e às suas próprias expectativas de bem-estar (ALMEIDA; GUTIERREZ; MARQUES, 2012). Não é possível simplificá-la em um único conceito, devido às diversas abordagens nas quais pode ser inserida. Entretanto, a partir da percepção do sujeito, é possível reunir elementos que atuem como seus indicadores objetivos e subjetivos (BARBOSA, 1998). Os indicadores objetivos são pautados em elementos quantificáveis e concretos, como alimentação, moradia, saúde, recursos financeiros, transporte, educação, etc. Já os indicadores subjetivos são os fatores emocionais, as expectativas, as oportunidades, as possibilidades, bem como as questões imensuráveis, como o prazer, a felicidade, a angústia e a tristeza (ALMEIDA; GUTIERREZ; MARQUES, 2012).

O presente artigo buscou investigar a qualidade de vida dos sujeitos com lesão medular mediante uma revisão sistemática. Os procedimentos metodológicos, descritos a seguir, têm como objetivo buscar na literatura pesquisas publicadas sobre o tema.

\section{Metodologia}

O presente artigo foi realizado através de uma revisão sistemática da literatura, conduzido conforme os métodos de Preferred Reporting Items for Systematic Reviews and Meta-Analyses - PRISMA (MOHER et al., 2009). Para identificar os abstracts sobre a qualidade de vida e a lesão medular, realizaram-se buscas nas bases de dados Sientific Eletronic Library Online (SciELO) e Coordenação de Aperfeiçoamento de Pessoal de Nível Superior (CAPES), de agosto de 2006 a agosto de 2019. Foram utilizadas as palavras-chave de busca: lesão medular, pessoas com deficiência e qualidade de vida.

Utilizaram-se os seguintes critérios de inclusão: (a) pesquisas exploratórias em pacientes com lesão medular adquirida por trauma, doença ou defeito congênito; (b) uso de instrumentos medidores quantitativos de qualidade de vida (WHOQOL-Bref elou SF-36); (c) artigos na língua portuguesa. Como critério de exclusão: (a) artigos sobre qualidade de vida que utilizaram metodologia qualitativa; (b) estudos comparativos; (c) revisões sistemáticas e integrativas.

A consulta nas bases de dados e a aplicação dos critérios de inclusão e exclusão viabilizou a leitura preliminar dos resumos e a identificação de duplicidade entre as bases, de modo a manter os estudos duplicados em uma única base. Revisaram-se os artigos selecionados e deles extraíram-se alguns itens para análise posterior dos textos completos, quais sejam: (1) Objetivos; (2) Método: amostra (número de participantes e instrumentos utilizados) e (3) resultados. 


\section{Resultados}

Finalizada a consulta nas bases de dados, com as palavras-chave supramencionadas, identificaram-se 80 estudos no total. Cinco estudos duplicados foram excluídos das bases de dados, totalizando 75 estudos. Em seguida, foram analisados os títulos e os resumos dos estudos, de forma a eliminar mais 53 estudos, por não se enquadrarem nos critérios de inclusão. Com isso, restaram 22 estudos para leitura e análise dos estudos completos. Após a leitura dos artigos na íntegra, foram aplicados os critérios de exclusão, a partir dos quais se retiraram 17 estudos por não aplicarem teste ou questionário que verifique a qualidade de vida do sujeito com lesão medular. Nessa etapa, também foram excluídos estudos que restringiram os questionários a familiares ou cuidadores da pessoa com lesão medular. Ao final, foram incluídos cinco artigos das buscas relatadas anteriormente. Na Figura 1, é apresentado o fluxograma com a síntese do processo de seleção dos artigos.

Figura 1: Fluxograma da síntese do processo de seleção dos artigos

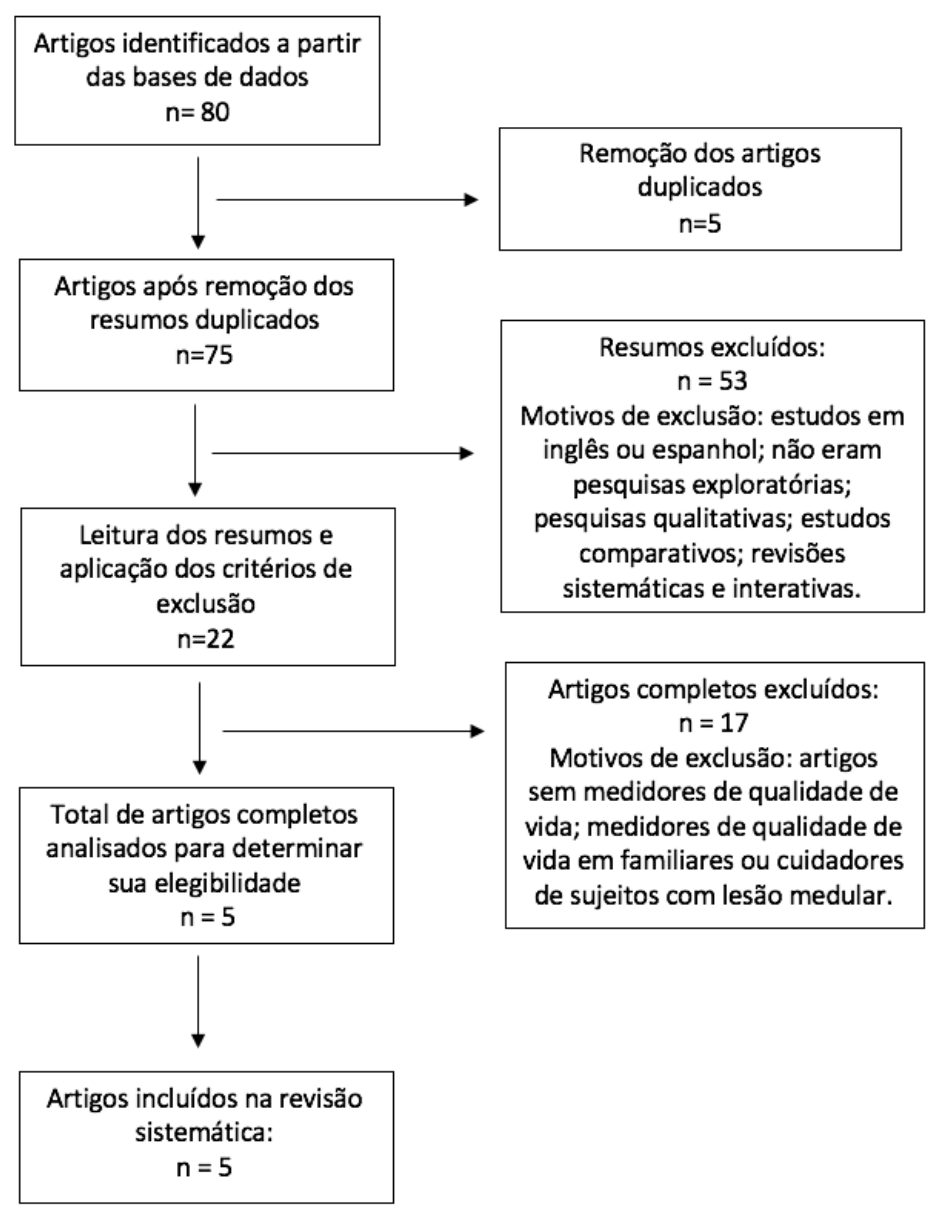

Fonte: Elaboração dos autores.

No quadro 1, são apresentados os artigos que, após minuciosa análise, foram selecionados para o desenvolvimento deste trabalho, dos quais são extraídas as seguintes Barbarói, Santa Cruz do Sul, n. 58, p.<141-153>,jan/jun 2021 
informações: os autores, o ano da publicação, os instrumentos utilizados para coleta dos dados, a amostra e os seus principais resultados. Entre os cinco artigos analisados, dois são do mesmo autor, os demais são de autores diversos. A busca enfocou estudos publicados em um período de dez anos, de 2006 a 2015. Observou-se uma variação de 30 a 111 participantes por amostra, considerando que em quatro, dos cinco artigos investigados, esse número era inferior a 50 participantes.

Em relação aos instrumentos utilizados, os pesquisadores aplicaram o WHOQOL-Bref em quatro artigos. Esse questionário é composto por 26 perguntas e tem como objetivo avaliar a qualidade de vida do sujeito em quatro domínios distintos, quais sejam: o Domínio Físico, o Domínio Psicológico, o Domínio das Relações Sociais e do Meio Ambiente. Uma pesquisadora recorreu a um questionário diverso e multidimensional, com idêntico objetivo, denominado "The MOS 36-item Short - Form Health Survey" (SF-36), o qual contém 36 itens e está dividido em oito domínios: a capacidade funcional, os aspectos físicos, a dor, o estado geral de saúde, a vitalidade, os aspectos sociais, os aspectos emocionais e a saúde mental.

Quadro 1:Artigos sobre Qualidade de Vida em sujeitos com lesão medular

\begin{tabular}{|c|c|c|c|c|}
\hline Autores & Ano & Instrumentos & Amostra & Resultados \\
\hline $\begin{array}{l}\text { Vall, Braga } \\
\text { e Almeida }\end{array}$ & 2006 & $S F-36$ & 32 & $\begin{array}{l}\text { Nesse estudo, observou-se que as variáveis } \\
\text { de capacidade funcional, aspectos físicos, } \\
\text { dor, estado geral de saúde, vitalidade, } \\
\text { saúde mental, aspectos sociais e } \\
\text { emocionais variaram entre } 43,36 \text { a } 63,16 \% \\
\text { nas porcentagens. O domínio de aspectos } \\
\text { sociais foi o mais comprometido nesse } \\
\text { estudo. A dor foi o menos comprometido. }\end{array}$ \\
\hline $\begin{array}{l}\text { Bampi, } \\
\text { Guilhem e } \\
\text { Lima }\end{array}$ & 2007 & $\begin{array}{c}\text { WHOQOL- } \\
\text { bref }\end{array}$ & 111 & $\begin{array}{l}\text { Os domínios que apresentaram os piores } \\
\text { escores de avaliação estavam relacionados } \\
\text { ao meio ambiente e à saúde física }(49,61 \% \\
\text { e } 50,26 \% \text {, respectivamente). Os melhores } \\
\text { escores estão relacionados à saúde } \\
\text { psicológica e às relações sociais. }\end{array}$ \\
\hline $\begin{array}{l}\text { França, } \\
\text { Coura, } \\
\text { França, } \\
\text { Basílio e } \\
\text { Souto }\end{array}$ & 2011 & $\begin{array}{c}\text { WHOQOL- } \\
\text { bref }\end{array}$ & 47 & $\begin{array}{l}\text { Domínio Físico ( } 58,59 \text { pontos; Mediana: } \\
62,55 \text {; Desvio padrão: 9,65). Domínio } \\
\text { Psicológico }(63,82 \text { pontos; Mediana: } 65,95 \text {; } \\
\text { Desvio padrão: } 7,64) \text {. Domínio das } \\
\text { Relações Sociais }(68,79 \text { pontos; Mediana: } \\
\text { 74,04; Desvio padrão: } 10,60) \text {. Domínio do } \\
\text { Meio Ambiente }(55,20 \text { pontos; Mediana: } \\
\text { 57,44; Desvio padrão: } 11,63) \text {. }\end{array}$ \\
\hline
\end{tabular}




\begin{tabular}{|c|c|c|c|c|}
\hline & & & & $\begin{array}{l}\text { A percepção dos participantes sobre a sua } \\
\text { QV alcançou um escore de } 61,70 \text { pontos, } \\
\text { sendo que os percentuais de } 44,7 \% \text { e de } \\
55,3 \% \text { denotaram a QV como boa e ruim. } \\
\text { No Domínio Físico, o escore foi de } 58,29 \\
\text { pontos, com o percentual de } 46,8 \% \\
\text { apontando satisfação e o de } 53,2 \% \\
\text { indicando insatisfação. No Domínio das } \\
\text { Relações Sociais, verificou-se a vida sexual } \\
\text { (44,7\%) como a de pior satisfação. As } \\
\text { relações pessoais (76,6\%), seguida pelo } \\
\text { apoio dos amigos (74,4\%), apresentaram } \\
\text { números mais positivos. O domínio que } \\
\text { apresentou a pior pontuação foi o do Meio } \\
\text { Ambiente. Todos os Domínios Físico, } \\
\text { Psicológico, das Relações Sociais e do } \\
\text { Meio Ambiente obtiveram escores baixos, } \\
\text { fato que pode ser explicado pelas várias } \\
\text { limitações que a lesão medular pode } \\
\text { acarretar. }\end{array}$ \\
\hline $\begin{array}{l}\text { França, } \\
\text { Coura, } \\
\text { Souza, } \\
\text { Almeida e } \\
\text { Pagliuca }\end{array}$ & 2013 & $\begin{array}{l}\text { WHOQOL- } \\
\text { bref }\end{array}$ & 47 & $\begin{array}{l}\text { A percepção dos entrevistados acerca da } \\
\text { sua QV nas duas últimas semanas foi } \\
\text { marcada pela satisfação ( } 46,8 \%) \text { ou pela } \\
\text { insatisfação }(53,2 \%) \text {. Domínios com } \\
\text { menores escores consistiram no Ambiental } \\
\text { (55,20 pontos) e no Físico ( } 58,59 \text { pontos). }\end{array}$ \\
\hline $\begin{array}{l}\text { Corrêa, } \\
\text { Lopes Neto } \\
\text { e Llapa- } \\
\text { Rodriguez }\end{array}$ & 2015 & $\begin{array}{l}\text { WHOQOL- } \\
\text { bref }\end{array}$ & 30 & $\begin{array}{l}\text { Dos } 30 \text { entrevistados, obtiveram-se os } \\
\text { seguintes resultados: } 20 \% \text { deles avaliou a } \\
\text { qualidade de vida geral como muito boa, } \\
36,7 \% \text { como boa e } 3,3 \% \text { como ruim; } 40 \% \\
\text { deles manifestou ter uma qualidade de vida } \\
\text { média. Em relação ao Domínio Físico, a } \\
\text { qualidade de vida foi avaliada como ruim } \\
\text { (30\%), média ( } 30 \%) \text {, boa ( } 30 \%) \text { e muito } \\
\text { boa ( } 10 \%) \text {. No Domínio Psicológico, ela } \\
\text { foi avaliada como boa ( } 56,7 \%) \text { média } \\
\text { ( } 20 \%) \text {, muito boa ( } 13,3 \%) \text { e ruim ( } 10 \%) \text {. } \\
\text { No Domínio das Relações Sociais, } 43,3 \% \text { a } \\
\text { avaliaram como média. }\end{array}$ \\
\hline
\end{tabular}

Fonte: Elaboração dos autores.

\section{Discussão}

O objetivo deste trabalho é fazer uma revisão sistemática em torno da qualidade de vida nos sujeitos com lesão medular. Os estudos trazem o conceito de qualidade de vida como algo com características variadas e peculiares, a qual compreende as capacidades comportamentais e cognitivas do sujeito, assim como seu bem-estar emocional. Portanto, é 
caracterizada com um conceito que compreende fatores físicos, psicológicos e sociais (CHAPPELL; WIRZ, 2003). Todos os artigos usaram como base o conceito de qualidade de vida da OMS, que é o mesmo usado para a população geral, não trazendo um conceito de qualidade de vida específico para sujeitos com lesão medular. Diante das bases de dados, o primeiro ponto a ser discutido são as poucas publicações sobre o tema nos últimos anos. O período no qual foram publicados os estudos foi de 2006 a 2015, mostrando que, nos últimos cinco anos, não foi realizada nenhuma publicação sobre a qualidade de vida em sujeitos com lesão medular.

O segundo ponto a ser discutido é a escassez de estudos publicados sobre o tema. Muitos estudos verificam a qualidade de vida no cuidador ou na família do sujeito com lesão medular, e não no sujeito em si. Dos cinco estudos investigados, dois utilizaram a mesma amostra, com objetivos semelhantes. Um deles buscou medir a qualidade de vida a partir da identificação dos domínios que a prejudicam (FRANÇA et al., 2013). Já o outro artigo dos autores teve como objetivo buscar quais fatores influenciam na qualidade de vida do sujeito com lesão medular (FRANÇA et al., 2011). Para esta revisão sistemática, pode-se dizer que há apenas quatro estudos, uma vez que os resultados de dois estudos encontrados são os mesmos, ainda que seus enfoques visassem a aspectos diferentes. Esse resultado mostra uma carência de trabalhos empíricos acerca do tema.

Outro aspecto a ser discutido são as pequenas amostras dos estudos realizados, dos quais apenas um apresentou uma amostra com número superior a 50 pessoas. A menor amostra corresponde a 30 sujeitos (CORREAA; LOPES NETO; LLAPA-RODRIGUEZ, 2015). Segundo Corrêa, Lopes Neto e Llapa-Rodriguez, que realizaram sua pesquisa em um Hospital universitário de Manaus, a amostra inicial do estudo era de 381 pessoas. Com a aplicação dos critérios de inclusão, apenas 194 pessoas tinham cadastro completo em seus prontuários. Após a realização de uma triagem, 45 sujeitos foram considerados aptos para responder à pesquisa. Todavia, 15 pessoas não aceitaram participar do estudo, o que culminou em uma amostra de 30 sujeitos.

Um ponto em comum em todas as pesquisas foi a predominância do sexo masculino. No estudo de Corrêa, Lopes Neto e Llapa-Rodriguez (2015), analisa-se 30 sujeitos com lesão medular, dentre os quais $88,3 \%$ são homens, o que se repete na pesquisa de Vall, Braga e Almeida (2006), na qual 29 dos 32 indivíduos estudados são do sexo masculino (90,6\%). Esse fato também é observado na investigação de França et al. (2013) e de França et al. (2011) que, a partir de uma amostra de 47 sujeitos, somam-se $43(91,5 \%)$ os homens que sofreram a lesão medular entre 13 e 30 anos; e de Bampi, Guilhem e Lima (2007), que registram 94 Barbarói, Santa Cruz do Sul, n. 58, p.<141-153>,jan/jun 2021 
homens $(84,7 \%)$ das 111 pessoas diagnosticadas com lesão medular, corroborando a hipótese de que o trauma é mais comum em pessoas do sexo masculino.

Em análise das 220 pessoas avaliadas nos cinco estudos, constata-se que as principais causas da lesão medular são por arma de fogo (72), acidentes de trânsito (68) e quedas (48). Segundo Bampi, Guilhem e Lima (2007), a principal causa de trauma são os acidentes de trânsito, os quais incluem carros, motos, atropelamentos e acidentes de bicicleta. Os autores aduzem, ainda, que o tipo de acidente mais comum ocorre pela marcante presença da violência urbana (BAMPI; GUILHEM; LIMA, 2007).

Outro aspecto importante desses estudos, associado diretamente à qualidade de vida, é a baixa renda dos entrevistados, dos quais a maioria (60\%) não recebia valor superior a dois salários mínimos no mês. Considera-se uma renda baixa diante dos grandes gastos com a saúde, principalmente com medicações, consultas médicas, fisioterapia, cuidadores, dentre outros custos. Segundo França et al. (2011), a hipossuficiência econômica acarreta a exclusão social e a falta de acesso às necessidades básicas. Para Vall, Braga e Almeida (2006), boa parte dos indivíduos estudados possui baixa escolaridade, devido às suas limitações motoras, sociais e financeiras, que representam um empecilho à sua instrução.

No Domínio Físico, Corrêa, Lopes Neto e Llapa-Rodriguez (2015) indicam que a qualidade de vida foi avaliada como ruim (30\%), média (30\%), boa (30\%) e muito boa (10\%), o que ficou bem dividido entre os participantes. A partir das análises realizadas em pesquisas americanas, Vall, Braga e Almeida (2006) relatam que quanto maior o comprometimento dedicado à lesão medular, menor será o escore no aspecto físico, o que resta demonstrado pelos resultados do seu estudo que, nesse domínio, apontam um comprometimento significativo na qualidade de vida.

Na pesquisa de Bampi, Guilhem e Lima (2007), no tocante ao referido domínio, os participantes relataram possuir energia para realizar as atividades do dia a dia e ter seu sono preservado. Contudo, estão insatisfeitos com a capacidade física para o trabalho e sua mobilidade muito reduzida. Na amostra de França et al. (2013), 20 participantes (42,5\%) afirmaram insatisfação com o desempenho das atividades da vida diária, o que resultou em maior dependência e na perda de autonomia. Conforme Venturini, Decesaro e Marcon (2006), o lesado medular apresenta diversos problemas orgânicos e físicos, tais como úlceras, infecções urinárias, espasticidade e deformidades. Em grande parte dos casos, eles ocasionam dificuldades e os tornam incapaz total ou parcialmente de movimentar-se, o que afeta diretamente sua saúde mental. 
No que concerne ao Domínio Psicológico, 56,7\% dos sujeitos avaliaram a qualidade de vida como boa, $20 \%$ avaliaram como média, 13,3\% como muito boa e 10\% como ruim (CORRÊA; LOPES NETO; LLAPA-RODRIGUEZ, 2015). Os achados no estudo Vall, Braga e Almeida (2006) apontam que os aspectos emocionais (59,38\%) e a saúde mental (62\%) também estão comprometidos, demostrando que a baixa autoestima gera menor satisfação com a vida e um sentimento maior de solidão, o que resulta em um desequilíbrio entre corpo, mente, espírito e ambiente, além de propiciar baixa qualidade de vida em todos os domínios. Bampi, Guilhem e Lima (2007) demonstram que os entrevistados se apegam a crenças pessoais, espiritualidade e religião, bem como aceitam sua aparência física, tentando manter a sua autoestima.

Na relação entre a depressão e a lesão medular, França et al. (2011) apontam que 25\% dos homens e $47 \%$ das mulheres do seu estudo apresentaram sintomas depressivos. Na amostra de Conceição et al. (2010), os índices de depressão são maiores logo após ocorrer a lesão medular. O lesionado medular deixa de realizar muitas atividades cotidianas pelas dificuldades enfrentadas e pelo período de transformação em sua vida. Ele poderá sofrer insônia, alterações no apetite, perda de peso, somados a muitos problemas com a autoimagem (ELLIOT, 2003; RADNITZ, 1994).

No Domínio Social, as relações pessoais e o apoio social foram fatores considerados importantes para inclusão. Por outro lado, a falta de oportunidades de lazer, os entraves no acesso ao ambiente físico e ao transporte, associados às questões financeiras, afetam diretamente a qualidade de vida do sujeito. Nos resultados apresentados por Vall, Braga e Almeida (2006), os aspectos sociais apresentam os índices mais baixos de avaliação (43,36\%) em relação aos outros domínios, o que não se repete nos demais estudos analisados.

A acessibilidade é primordial para que o sujeito com lesão medular consiga transitar de forma autônoma (CORREAA; LOPES NETO; LLAPA-RODRIGUEZ, 2015). Nos achados de Bampi, Guilhem e Lima (2007), fatores ambientais comprometeram a qualidade de vida, sobretudo na participação social, pois os indivíduos afetados apresentavam insatisfação com a vida após a lesão, relacionando-a com as desvantagens enfrentadas. Para Corrêa, Lopes Neto e Llapa-Rodriguez (2015), as atividades que os indivíduos realizavam em casa (tarefas cotidianas) foram consideradas satisfatórias, de modo diverso das atividades que aconteciam fora da residência, como o acesso a serviços, transporte e lazer, consideradas insatisfatórias.

De forma geral, as pesquisas desta revisão sistemática apontam que o sujeito com lesão medular percebe a qualidade de vida como boa, em geral, e satisfatória, no âmbito da saúde. Os resultados da maior parte dos estudos indicam que os menores índices de qualidade 
de vida observados estão no Domínio do Meio Ambiente e Físico, em decorrência da acessibilidade, o que acaba por comprometer os demais domínios.

\title{
6. Conclusão
}

O objetivo desse estudo, foi realizar uma revisão sistemática da literatura sobre a qualidade de vida do sujeito com lesão medular, permitindo conhecer a produção científica publicada no período de 2006 a 2019, nas bases de dados CAPES e Scielo. Observou-se, nesse estudo, que são escassas as pesquisas publicadas sobre a qualidade de vida em lesados medulares, bem como é pequena a quantidade de sujeitos que integram as amostras.

Pontua-se que os domínios físico e ambiental de qualidade de vida mostraram-se os mais comprometidos em quase todos os artigos aqui citados. As condições físicas e ambientais se correlacionam, o que compromete domínios relacionados à locomoção, à acessibilidade no trabalho, ao transporte, à saúde e ao lazer, e afeta a socialização. Apesar disso, a qualidade de vida (em geral) do sujeito com lesão medular se mostrou boa e satisfatória em diversas pesquisas.

Nota-se que o instrumento utilizado nas pesquisas, por mais que reconhecido e validado, torna-se muito genérico, razão pela qual se recomenda o desenvolvimento de uma ferramenta mais específica para a avaliação da qualidade de vida em lesados medulares. A atualização das pesquisas também se faz necessária para avaliar as mudanças que ocorrem na vida dos sujeitos afetados por essas lesões e como eles percebem sua qualidade de vida. Espera-se que o resultado dessa investigação contribua no planejamento de ações voltadas às pessoas com lesão medular, ampliando sua acessibilidade, de modo a aumentar sua autonomia e sua independência social e profissional.

\section{QUALITY OF LIFE IN SUBJECTS WITH SPINAL INJURY: A SYSTEMATIC REVIEW}

\begin{abstract}
The aim of this study is to analyze and investigate the quality of life in people with spinal cord injury, through a systematic review. The main studies published in the period 2006 to 2019 were searched in the CAPES, Scielo. All the studies used quantitative measure about quality of life, which were the WHOQOL-Bref and the SF-36. The results generally showed that the subject with spinal cord injury evaluates their quality of life as good and their health in a general satisfactory manner. The physical and environmental domains were the most cited domains in the researches as a negative factor, being related to the issue of accessibility. It was found that future studies are extremely important to understand the quality of life of these individuals, as well as for the improvement of public policies for the question of accessibility and autonomy of these subjects.
\end{abstract}

Barbarói, Santa Cruz do Sul, n. 58, p.<141-153>, jan/jun 2021 
Keywords: Spinal cord injury. Disabled persons. Quality of life.

\section{REFERÊNCIAS}

ALMEIDA, M. A. B.; GUTIERREZ, G. L.; MARQUES, R. Qualidade de vida: definição, conceitos e interfaces com outras áreas de pesquisa. São Paulo: EACH/USP, 2012.

BAMPI, L. N. da S.; GUILHEM, D.; LIMA, D. D. Qualidade de vida em pessoas com lesão medular traumática: um estudo com o WHOQOL-bref. Revista Brasileira de

Epidemiologia, São Paulo, v. 11, n. 1, p. 67-77, mar. 2008. Disponível em:

http://www.scielo.br/scielo.php?script=sci_arttext\&pid=S1415-

790X2008000100006\&lng=en\&nrm=iso. Acesso em: 18 nov. 2020.

BARBOSA, S. R. da C. S. Qualidade de vida e ambiente: uma temática em construção. In:

BARBOSA, S. R. da C. S. (Org.). A temática ambiental e a pluralidade do Ciclo de

Seminários do NEPAM. Campinas: UNICAMP; NEPAM, 1998. p. 401- 423.

BERTO, C. D; BARRETO, D. B. M. Pessoas com lesão medular traumática: as alterações biopsicossociais e as expectativas vividas. Unoesc \& Ciência - ACHS, Joaçaba, v. 2, n. 2, p. 174-183, jul./dez. 2011. Disponível em:

http://editora.unoesc.edu.br/index.php/achs/article/view/718/pdf_219. Acesso em: 15 de nov. de 2020.

CAMPOS, et al. Epidemiologia do traumatismo da coluna vertebral. Revista do Colégio Brasileiro de Cirurgiões, Rio de Janeiro, v. 35, n. 2, p. 88-93, abr. 2008. Disponível em: https://www.scielo.br/scielo.php?pid=S0100-

69912008000200005\&script=sci_abstract\&tlng=pt. Acesso em: 5 dez. 2020.

CASALIS, M. E. P. Lesão Medular. In: TEIXEIRA, E. et al. Terapia ocupacional na reabilitação física. São Paulo: Roca, 2003. p. 41-61.

CHAPPELL, P; WIRZ, S. Quality of life following spinal cord injury for 20-40 year old males living in Sri Lanka. Asia Pacific Disability Rehabilitation Journal, Tóquio, v. 14, n. 2, p. 162-178, 2003. Disponível em:

https://www.researchgate.net/publication/228496263_Quality_of_life_following_spinal_cord _injury_for_20-40_year_old_males_living_in_Sri_Lanka. Acesso em: 4 dez. 2020.

CONCEIÇÃO, M. I. G. et al. Avaliação da depressão em pacientes com lesão medular.

Revista Brasileira de Terapia Comportamental e Cognitiva, São Paulo, v. 12, n. 1-2, p. 43-59, jun. 2010. Disponível em:

http://pepsic.bvsalud.org/scielo.php?script=sci_arttext\&pid=S1517-

55452010000100003\&lng=pt\&nrm=iso. Acesso em: 8 dez. 2020.

CORRÊA, L. da S.; LOPES NETO, D.; LLAPA-RODRIGUEZ, E. O. Qualidade de vida de pessoas com lesão medular traumática. Revista Cogitare Enfermagem, Curitiba, v. 20, n. 4, p. 695-700, out./dez. 2015. Disponível em: https://revistas.ufpr.br/cogitare/article/view/41403. Acesso em: 7 dez. 2020. 
COURA, A. S. et al. Functional disability of adult individuals with spinal cord injury and its association with socio-demographic characteristics. Revista Latino-Americana de

Enfermagem, Ribeirão preto, v. 20, n. 1, p. 84-92, 2012. Disponível em:

https://www.scielo.br/scielo.php?script=sci_arttext\&pid=S0104-11692012000100012. Acesso em: 02 dez. 2020.

ELLIOT, T. R. Depressão após lesão da medula espinhal. Revista Brasileira de Psiquiatria, São Paulo, v. 25, n. 1, p. 51-54, 2003.

FLECK, M. P. A. et al. Aplicação da versão em português do instrumento abreviado de avaliação da qualidade de vida "WHOQOL-bref". Revista de Saúde Pública, São Paulo, v. 34, n. 2, p. 178-183, 2000. Disponível em:

https://www.scielo.br/scielo.php?script=sci_arttext\&pid=S0034-89102000000200012. Acesso em: 19 maio 2020.

FRANÇA, I. S. X. de et al. Qualidade de vida em pacientes com lesão medular. Revista Gaúcha de Enfermagem, Porto Alegre, v. 34, n. 1, p. 155-163, mar. 2013. Disponível em: http://www.scielo.br/scielo.php?script=sci_arttext\&pid=S198314472013000100020\&lng=en\&nrm=iso. Acesso em: 08 nov. 2020.

FRANÇA, I. S. X. de et al. Qualidade de vida de adultos com lesão medular: um estudo com WHOQOL-bref. Revista da Escola de Enfermagem da USP, São Paulo, v. 45, n. 6, p. 13641371, dez. 2011. Disponível em:

http://www.scielo.br/scielo.php?script=sci_arttext\&pid=S0080-

62342011000600013\&lng=en\&nrm=iso. Acesso em: 18 nov. 2020.

MANZINI, E. J. (Org.). Inclusão e acessibilidade. Marilia: ABPEE, 2006.

MEDOLA, F. et al. Avaliação do alcance funcional de indivíduos com lesão medular espinhal usuários de cadeira de rodas. Revista Movimenta, Anápolis, v. 2, n. 1, p. 12-16, 2009. Disponível em: https://www.revista.ueg.br/index.php/movimenta/article/view/7202. Acesso em: 07 dez. 2020.

MINAYO, M. C. de S.; HARTZ, Z. M. de A.; BUSS, P. M. Qualidade de vida e saúde: um debate necessário. Ciência \& Saúde coletiva, Rio de Janeiro, v. 5, n. 1, p. 7-18, 2000.

Disponível em: https://www.scielo.br/scielo.php?pid=S1413-

$81232000000100002 \&$ script=sci_abstract\&tlng=pt. Acesso em: 04 dez. 2020.

MOHER, D. et al. Reprint-preferred reporting items for systematic reviews and metaanalyses: the PRISMA statement. Physical Therapy, Alexandria, v. 89, n. 9, p. 873-880, 2009.

MOREIRA, W. W. Qualidade de vida: complexidade e educação. Campinas: Papirus, 2001.

RADNITZ, C. L. et al. Spinal cord injury: a search for determinants of depression two years after the event. British Journal of Clinical Psychology, [s.l.], v. 33, n. 2, p. 221-230, 1994. Barbarói, Santa Cruz do Sul, n. 58, p.<141-153>, jan/jun 2021 
VALL, J.; BRAGA, V. A. B.; ALMEIDA, P. C. de. Estudo da qualidade de vida em pessoas com lesão medular traumática. Arquivos de Neuro-Psiquiatria, São Paulo, v. 64, n. 2b, p. 451-455, Jun. 2006. Disponível em:

http://www.scielo.br/scielo.php?script=sci_arttext\&pid=S0004-

282X2006000300019\&lng=en\&nrm=iso. Acesso em: 5 dez. 2020.

VENTURINI, D.; DECESARO, M., MARCON, S. Conhecendo a história e as condições de vida de indivíduos com lesão medular. Revista Gaúcha de Enfermagem, Porto Alegre, v. 27, n. 2, p. 219-229, 2006.

Data de recebimento: $21 / 09 / 2020$

Data de aceite: 03/01/2021

\section{Sobre os autores:}

Carolina Antunes é Mestranda em Diversidade Cultural e Inclusão Social - Universidade Feevale (Bolsista CAPES); Bacharel em Psicologia; Psicóloga Cognitivo-Comportamental; Especialista em Psicologia Infantil. Endereço Eletrônico: carolinaa.antunes@ gmail.com

Marcus Levi Lopes Barbosa é Psicólogo. Doutor em Ciências do Movimento Humano. Coordenador do Mestrado Acadêmico em Psicologia. Professor adjunto do Curso de Psicologia, Mestrado Acadêmico em Psicologia e do Programa de Pós-Graduação em Diversidade Cultural e Inclusão Social da Universidade Feevale. Endereço Eletrônico: marcusl@feevale.br

Gustavo Roese Sanfelice possui graduação em Educação Física pela Universidade Federal de Santa Maria (2001); mestrado em Ciência do Movimento Humano pela Universidade Federal de Santa Maria (2002) e doutorado em Ciências da Comunicação/Universidade do Vale do Rio dos Sinos/Unisinos (2007). Atualmente é professor Titular da Universidade Feevale. Coordenador do Programa de Pós-Graduação em Diversidade Cultural e Inclusão Social/Feevale. Endereço Eletrônico: sanfeliceg@ feevale.br

Jacinta Sidegum Renner é Doutora e mestre em Engenharia de Produção (UFRGS) Fisioterapeuta e especialista em Saúde do Trabalhador. Professora e pesquisadora do PPG em Diversidade Cultural e Inclusão Social da Universidade Feevale. Endereço Eletrônico: jacinta@feevale.br

Gabriel Feiten possui graduação em Psicologia - Faculdades Integradas de Taquara (2010). Pós-graduação em Psicologia do Esporte e Treinamento Físico- Universidade Feevale (2012) e é Mestrando no Programa de Diversidade Cultural e Inclusão Social pela mesma instituição. Atualmente é psicólogo na Prefeitura Municipal de Três Coroas/RS. Endereço Eletrônico: psicofeiten@gmail.com 